\title{
Circuits Design and Nano-Structured Electrodes for Drugs Monitoring in Personalized Therapy
}

\author{
Sandro Carrara*, Andrea Cavallini, \\ Giovanni De Micheli \\ Swiss Federal Institute of Technology - Lausanne (EPFL) \\ CH-1015 Lausanne (CH) - * sandro.carrara@epfl.ch \\ Jacopo Olivo, Luca Benini \\ D.E.I.S. Department - Bologna University (IT)
}

\author{
Victoria V. Shumyantseva, \\ Alexander I. Archakov \\ Institute of Biomedical Chemistry \\ Russian Academy of Biomedical Science - Moscow (RU)
}

\begin{abstract}
Drug development and personalized therapy require accurate and frequent monitoring of the metabolic response by living tissues to treatments. In case of high risk side effects, e.g. therapies with interfering anti-cancer molecule cocktails, direct monitoring of the patient's drugs metabolism is essential as the metabolic pathways efficacy is highly variable on a patient-bypatient basis. Currently, there are no fully mature point-of-care bio-sensing systems for drugs metabolism monitoring directly in blood. The aim of the paper is to investigate solutions to develop point-of-care systems for drugs monitoring in personalized therapy. P450 enzymes are the considered probe molecules as they are key proteins directly involved in drugs metabolism of humans. Sensitivity improvement is ensured by means of enzyme integration onto electrodes structured with carbon nanotubes. Component Off-The-Shelf (COTS) based circuits design is investigated toward bio-chip development. Results show that the proposed circuitry is suitable for the aim and confirm that nanotubes are detection enhancers.
\end{abstract}

\section{INTRODUCTION}

Despite increased expenditure for drug development, sophisticated high-throughput techniques and apparently infinite possibilities offered by the rapidly expanding knowledge in genetics, still most effective drug therapies for major diseases provide benefit only to a fraction of patients [1]. This fraction is typically in the 20 to $50 \%$ range, while approximately $7 \%$ of hospitalized patients have serious adverse drug reactions [2]. This applies especially for main killers in Western countries, i.e. heart diseases and cancer. While ten years ago treatment of patients was thought to be based best on the mean results of randomized clinical trials and resulting guidelines (evidence based medicine), currently it is being increasingly recognized that any drug therapy needs to be selected and modified according to the individual patient ("individualization" or "personalization"). [3, 4].

It has been known for decades that genetic polymorphism in P450 2D6 (a central enzyme in human metabolism) causes overdosing in "poor metabolizers" and under-dosing in "ultrafast metabolizers". This is important e.g. in the treatment of chronic heart failure, hypertension (metoprolol), cancer (tamoxifen), pain, depression, or schizophrenia. However, the currently availability of tools for acquiring this knowledge is very limited and it is a major weakness of the concept of personalized medicine. Therefore, the development of Biochip array platforms based on P450 proteins to detect the metabolism of pharmacological compounds is required to pursuit personalization in drug therapies.

Nanostructures, such as metal nanoparticles and/or carbon nanotubes, may be used to improve the sensitivity of such P450-based biosensors. It has been shown in 2005 that P450 $11 \mathrm{~A} 1$ activity is highly enhanced when the cytochrome is immobilized onto screen-printed electrodes modified by gold nanoparticles [5]. $100 \mu \mathrm{M}$ of cholesterol produced a current signal of a few $\mu \mathrm{A}$ when using riboflavin as molecular mediator between P450 11A1 and rhodium-graphite screenprinted electrodes with an area of $20 \mathrm{~mm}^{2}$ [6]. On the other hand, a $10 \mu \mathrm{M}$ concentration produced a current signal of more than $1 \mu \mathrm{A}$ when using screen-printed electrodes of the same area but modified by gold nanoparticles [5]. More recently, further improvement in the sensitivity of cholesterol biosensor was demonstrated with nano-structuring by means of carbon nanotubes [7]. In this paper, the viability of nanostructured electrodes functionalized with P450 enzymes has been investigated in order to evaluate the feasibility of an integrated bio-chip solution. Circuit architecture for electrochemical measurements has been designed, realized, and tested.

\section{CIRCUIT DEVELOPMENT}

A Printed Circuit Board (PCB) prototype has been realized using off-the-shelf components in order to investigate the feasibility of a fully integrable technology for electrochemical detection in drugs monitoring.

\section{A. Specifications}

Literature analysis of voltammetries with cytochrome P450 as detection probe [8-13] provided the following specifications for the circuit which query a three electrodes configuration sensing spot:

- At working electrode: must be able to generate a triangular wave fully customizable in amplitude range $(-1000 \div 1000$ $\mathrm{mV})$ and in scan rate $(10 \div 100 \mathrm{mV} / \mathrm{s})$. To realize a 
Differential Pulse Voltammetry (DPV), thus to generate the sum of a triangular wave with a square one. To apply a constant potential within the range from -1000 to 1000 $\mathrm{mV}$ ). To also allow Chronoamperometry (CA) experiments.

- At reference electrode: to read the potential of the solution in the range $-1000 \div 1000 \mathrm{mV}$.

- At counter electrode: to read the current flowing through the solution (in the range $-1000 \div 1000 \mu \mathrm{A}$ ) due to the oxidation and reduction processes.

\section{B. Architecture}

The entire system consists of the electronic board for generation and acquisition of signals and by a remote PC. They communicate by using UART protocol to set the signals to be generated and to analyze signals detected on the sample. The board architecture is shown in Figure 1. The single components are $\mathrm{R} 1=48 \mathrm{~K} \Omega, \mathrm{R} 2=47 \mathrm{~K} \Omega, \mathrm{R} 3=250 \mathrm{~K} \Omega, \mathrm{R} 4=100 \mathrm{~K} \Omega, \mathrm{R} 5$ in the range from 1 to $10 \mathrm{~K} \Omega \mathrm{R} 6$ in the range from 2,5 to 250 $\mathrm{k} \Omega$. The two IC are U1 TLC2274AID (from Texas Instruments), U2 OP497GS (Analog Devices). Finally, the capacitors are $\mathrm{C} 1=7,5 \mathrm{nF}, \mathrm{C} 2=10 \mathrm{nF}$. This specific solution is able to scan a single site, but it was chosen to be realized as integrated circuit, and easily upgraded to a multiple read.

The triangular wave is obtained by isolating the average value of a square wave with a duty cycle changing linearly in time. This result is obtained using a Bessel low-pass filter to keep only the DC value of the square wave. The solution gives a clean wave by using a single bit, and generates triangular signals with multiple frequencies with a low hardware effort. The microcontroller generates the square wave to be filtered and the square wave to be eventually summed for DPV. It also reads converts and sends the acquired signals to the remote PC for off-line analysis. The other logic on board has the purpose to shift and adapt the signals in the appropriate ranges.

The entire architecture is easy to be realized in an integrated circuit. The low-pass Bessel filter is the only component requiring particular attention due to capacitors in the order of $\mathrm{nF}$. Possible solutions of this problem are the use of switched capacitors or digital filters.

\section{MATERIALS AND METHODS}

\section{A. Materials}

Carbon paste screen printed electrodes were purchased from Dropsens (model DRP-110). The electrodes were made of a graphite working electrode (area equal to $13 \mathrm{~mm}^{2}$ ), a graphite counter electrode and an $\mathrm{Ag} / \mathrm{AgCl}$ reference electrode. The total area of the cell is $22 \mathrm{~mm}^{2}$. Cytochrome $\mathrm{P} 450$ 2B4 was isolated and purified from the microsomal fraction of rabbit [14]. It was diluted in PBS buffer $50 \mathrm{mM}$ at pH 7.4 with $20 \%$ glycerol content. Benzphetamine drug (from Sigma) was diluted in PBS pH 7.4 in aliquots of $50 \mathrm{nM}, 5 \mu \mathrm{M}$, $500 \mu \mathrm{M}, 50 \mathrm{mM}$. Single wall carbon nanotubes (SWCNT, diameter 1-2 nm, length $30 \mu \mathrm{m}, \mathrm{COOH}$ content $2,73 \%$ ) were purchased in powder ( $90 \%$ purity) from MKnano (Canada), diluted in chloroform to the concentration of $1 \mathrm{mg} / \mathrm{mL}$ [7].

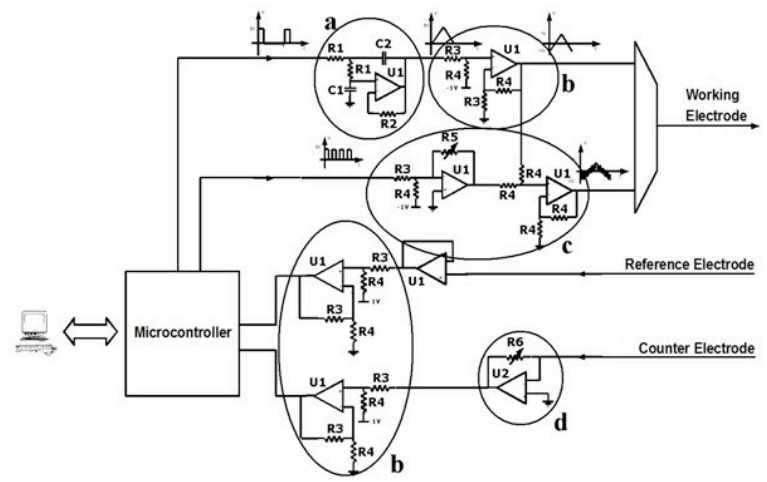

Fig. 1: Schematic of the PCB: a) Bessel Filter; b) Shifter \& Range Adapters; c) Analogic Adder; d)I/V Converter.

Samples were then sonicated for 20 minutes in order to form a homogeneous suspension.

\section{B. Preparation of enzyme electrode}

The working electrode was prepared in a two steps procedure [7]: $10 \mu \mathrm{L}$ of SWCNT solution were drop casted onto the working electrode and allowed to dry, then $3 \mu \mathrm{L}$ of P450 2B4 solution were spread onto the nanostructured working electrode and incubated at $4^{\circ} \mathrm{C}$ overnight.

\section{Electrochemical measurements}

The electrochemical response of P450 2B4 immobilized onto nano-structured electrodes was investigated by cyclic voltammetry under aerobic conditions. Voltammograms were acquired by using a Versastat 3 potentiostat (Princeton Applied Technologies). The electrodes were covered with 100 $\mu \mathrm{L}$ of PBS $100 \mathrm{mM}$ at $\mathrm{pH} 7.4$, or with the same volumes of PBS added to $1 \mu \mathrm{L}$ of Benzphetamine samples. The potential was swept in the range from -600 to $+100 \mathrm{mV}$ vs $\mathrm{Ag} / \mathrm{AgCl}$ using a scan rate of $100 \mathrm{mV} / \mathrm{sec}$.

\section{EXPERIMENTAL RESULTS}

\section{A. PCB test for electrochemical detection}

The board has been tested with the standard cyclic voltammetry of Potassium Ferrocyanide. $\left.\mathrm{K}_{4}\left[\mathrm{Fe}(\mathrm{CN})_{6}\right)\right] \cdot 3 \mathrm{H}_{2} \mathrm{O}$, at concentration of $30 \mathrm{mM}$, was diluted in PBS at $100 \mathrm{mM}$ and $\mathrm{pH}$ 7.4. Voltammograms reported in Figure 2 were acquired by using screen printed electrodes nano-structured with SWCNT and a scan rate of $100 \mathrm{mV} / \mathrm{s}$.

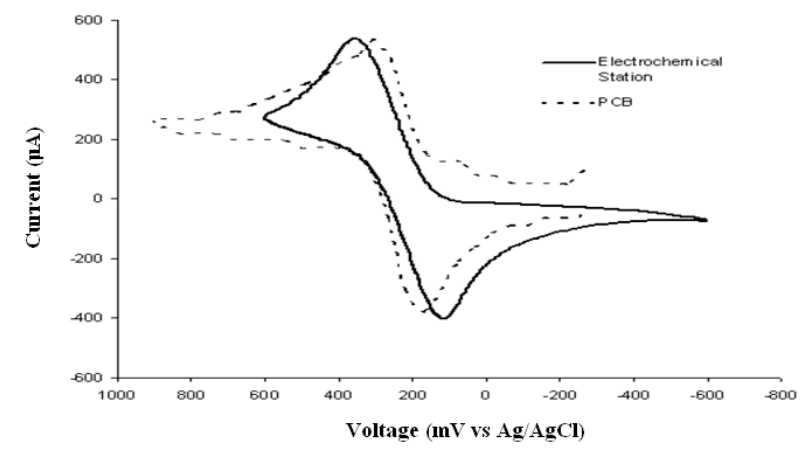

Fig. 2: Voltammogram acquired with the developed board in comparison with that from a lab station. 


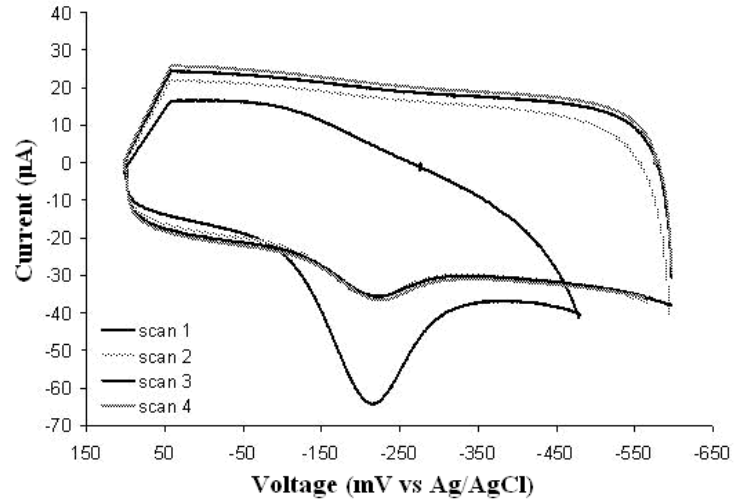

Fig. 3: Cyclic voltammograms of screen-printed graphite electrodes functionalized with SWCNT. Acquisitions were done by using a lab potentiostat. Scan 1 is different as due to ions charging.

Curves shape unequivocally confirms a good performance of the architecture proposed in Figure 1 in comparison with that of an electrochemical lab station. Data compare quite well with those published in literature on the same redox reaction [15]. A low $\mathrm{S} / \mathrm{N}$ ratio is also clearly evident.

\section{B. P450 $2 B 4$ response onto SWCNT electrodes}

The peaks obtained in scans of SWCNT alone and SWCNT + P450 2B4 were analyzed. Figure 3 shows the first 4 scans on PBS of an electrode nano-structured with SWCNT. The peak at $-220 \mathrm{mV}$ is due to the oxygen moieties derived from CNT $[16,17]$. During the scans, we noticed an increase of the total voltammogram area and a decrease of the peak area. The capacitive baseline current was subtracted and Gaussian decomposition was used to define each contribution into peak shape. Figure 4 shows the peak acquired on cytochrome immobilized onto SWCNT. Peak shape is asymmetric and it is described using two different Gaussian curves. In case of SWCNT alone, data are well described by a single Gaussian curve. In case of both, the smaller peak has its center exactly positioned in the redox potential of P450 2B4 ($350 \mathrm{mV}$ ) [14]. The Gaussian decomposition of SWCNT/P450 2B4 peak strongly confirms similar features observed by using P450 11A1 and multi walled carbon nanotubes [7].

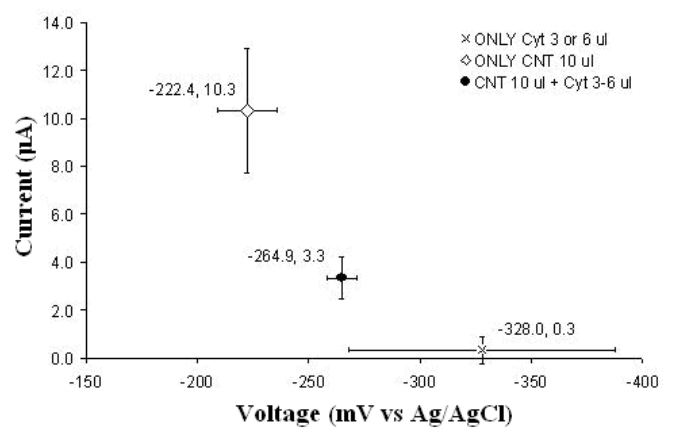

Fig. 5: Peak current versus potential after Cytochrome P450, SWCNT, SWCNT and P450 drop casted. Values were obtained averaging the last three scans of three different electrodes.

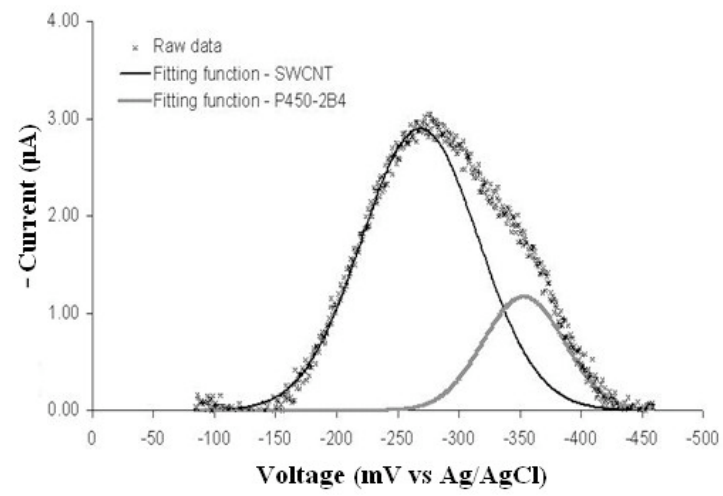

Fig. 4: Gaussian decomposition of acquired peak. with SWCNT and $\mathrm{P} 450-2 \mathrm{~B} 4$. Acquisitions were done by using a lab potentiostat.

Both SWCNT nano-structuring and P450 functionalization cause a huge increase in the total area of the voltammograms. (data not shown). Drop cast SWCNT behave like a 3D porous structure in which solution ions can penetrate [18]. It is also interesting to note that peak shifts towards more negative potentials after P450 2B4 functionalization, and the current has a severe decrease (Figure 5). This means that the electrolyte is less accessible to SWCNTs and more energy is required for electrons transfer. However, electrodes with Cytochrome and SWCNT present less energy for an improved electron transfer in comparison with electrodes functionalized with only P450 2B4. The use of CNT seems to confine peak positions into a narrowed potential window improving reproducibility. Multiple voltammetric scans force ions inside the SWCNT matrix and assist the electrical double layer formation around the SWCNT walls, as shown by the fist scan in Figure 3 which differs from others. The drop casting of proteins further alters charge balance because new charges are introduced onto the surface [19]. Capacitance increases till equilibrated double layer prevents further charged species to reach the surface. In our experiments we found that the total charge increases linearly with increasing volumes of SWCNT, but after the addiction of $\mathrm{P} 450$ this linearity is no more respected.

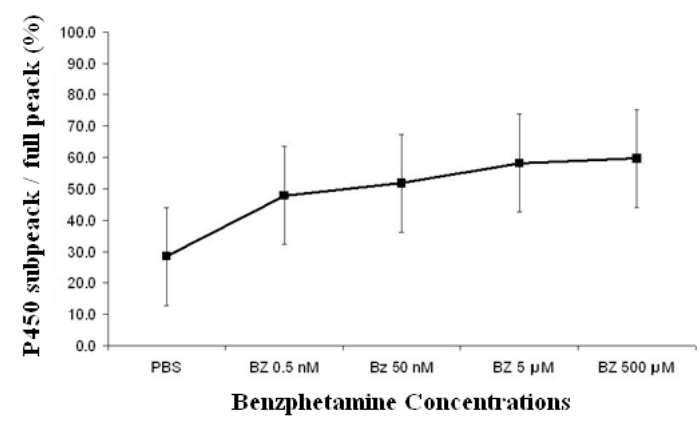

Fig. 6: Ratio between P450 current sub-peak and CNT peak with respect to various benzphetamine addictions. 
Total capacitance saturation is reached in case of cytochromes and SWCNT (data not shown). This capacitive current limits the faradic current due to CNT oxygen and cytochrome.

\section{Benzphetamine Detection}

The voltammetric response to increasing concentrations of Benzphetamine (BZ) was also measured. Drops of $1 \mu \mathrm{L}$ of BZ solution were progressively added to the $100 \mu \mathrm{L}$ drop of PBS covering the electrode. Solutions with concentrations of 0.5 $\mathrm{nM}, 50 \mathrm{nM}, 5 \mathrm{uM}, 500 \mathrm{uM}$ of BZ were used. No peak current increase was clearly observed after $\mathrm{BZ}$ addiction, but the ratio of cytochrome sub-peak to the recorded voltammetric peak increase when more drug is added (Figure 6). This demonstrates the possibility to detect BZ. Moreover, the P450 sub-peak shifted in presence of BZ accordingly with literature[14].

\section{CONClusions}

In this paper, the feasibility of on-chip point-of-care devices for drugs monitoring in personalized therapies has been investigated. Data from electrochemical acquisition on carbon based screen printed electrodes have been shown. The electrodes were nano-structured by using Single Walled Carbon Nanotubes with diameter of $2 \mathrm{~nm}$ and presenting $\mathrm{COOH}$ functional groups. The nano-structured electrodes were functionalized by solution casting of P450 2B4 enzyme.

The redox activity of the protein $\mathrm{P} 450$ 2B 4 onto these electrodes was demonstrated by using a lab electrochemical station. Detection of a common drug (Benzphetamine) was proved as well. Other pharmacological compounds, such as aminopyrine, 7-pentoxyresorufin, and lanosterol, are detectable by using the same P450 2B4 [14]. Moreover, different P450 proteins may detect other drugs commonly used in pharmacological therapy [20]. The architecture of a detection circuit was designed, prototyped onto $\mathrm{PCB}$, and successfully tested. Hence, the first step to realize point-of-care bio-chip to monitor drugs metabolism is overcome. Future works will focus on the fabrication and test of P450s based array for multipanel drugs detection and on VLSI fabrication and test of all the electronic circuitry required for the electrochemical detection as well as for multiplexing, storing and communication.

\section{ACKNOWLEDGMENT}

S.C. thanks Uwe Furh for highly useful discussions on personalized therapies and electrochemistry of P450. J.O. thanks Yuksel Temiz, Augusto Pieracci, Omar Cafini, and Frank Gürkaynak for their suggestions in circuit design and software implementation.

\section{REFERENCES}

1. Uwe Furh, personal communication

2. Lazarou J., Pomeranz B.H., Corey P.N. Incidence of adverse drug reactions in hospitalized patients. A meta-analysis of prospective studies. JAMA. 1998;279:1200-5

3. Stephen T. Turner; Gary L. Schwartz; Eric Boerwinkle, Personalized Medicine for High Blood Pressure, Hypertension. 2007;50:1-5;

4. Daly AK. Individualized drug therapy, Curr Opin Drug Discov Devel. 2007;10:29-36

5. Victoria V. Shumyantseva, Sandro Carrara, Valter Bavastrello, Jason D. Riley, Tatiana V. Bulko, Sergei A. Usanov, Claudio Nicolini and Alexander I. Archakov: "Direct electron transfer between cytochrome P450scc and screen-printed rhodium graphite electrode modified with gold nanoparticles", Biosensors \& Bioelectronics, 21, 217-222, (2005)

6. Shumyantseva V, De Luca G, Bulko T., Carrara S, Nicolini C, Usanov S.A., Archakov A: "Cholesterol amperometric biosensor based on cytochrome P450scc”, Biosensor \& Bioelectronics, 19, 971-976, (2004).

7. Sandro Carrara, Victoria V. Shumyantseva, Alexander I. Archakov, Bruno Samorì, Screen-Printed Electrodes based on Carbon Nanotubes and Cytochrome P450scc for Highly-Sensitive Cholesterol Biosensors, Biosensors and Bioelectronics, 24(2008) 148-150.

8. S. Joseph, J.F. Rusling, Y.M. Lvov, T. Friedberg, U. Fuhr, "An amperometric biosensor with human CYP3A4 as a novel drug screening tool", Biochemical Pharmacology, Vol. 65, Nr. 11, 2003, pp. 1817-1826.

9. S. Liu, L. Peng, X. Yang, Y. Wu, L. He, "Electrochemistry of cytochrome $\mathrm{P} 450$ enzyme on nanoparticle-containing membrane-coated electrode and its applications for drug sensing", Analytical Biochemistry 375, 2008, pp. 209-216.

10. L. Peng, X. Yang, Q. Zhang, S. Liu, "Electrochemistry of Cytochrome P450 2B6 on electrodes modified with zirconium dioxide nanoparticles and platin components", Electroanalysis, Vol. 20, Issue 7, pp. 803-807.

11. E. Iwuoha, R. Ngece, M. Klink, P. Baker, "Amperometric response of CYP2D6 drug metabolism nanobiosensor for sertraline; a selective serotonin reuptake inhibitor", IET Nanobiotechnology, Vol. 1, Issue 4, 2007, pp 62-67

12. C. Estavillo, Z. Lu, I. Jansson, J.B. Schenkman, J.F. Rusling, "Epoxidation of styrene by human cyt P450 1A2 by thin film electrolysis and peroxide activation conpared to solution reactions", Biophysical Chemistry, Vol. 104, Issue 1, 2003, pp.291-296.

13. D.L. Johnson, B.C. Lewis, D.J. Elliot, J.O. Miners, L.L. Martin, "Electrochemical characterisation of the human cytochrome P450 CYP2C9", Biochemical Pharmacology, Vol. 69, Issue 10, 2005, pp.1533-1541

14. V.V. Shumyantseva, T.V. Bulko, Yu.O. Rudakov, G.P. Kuznetsova, N.F. Samenkova, A.V. Lisitsa, I.I. Karuzina, A.I. Archakov, Electrochemical properties of cytochroms P450 using nanostructured electrodes: Direct electron transfer and electro catalysis, Journal of Inorganic Biochemistry, 101 (2007) 859-865

15. C. Berggren, P. Stålhandske, J. Brundell, G. Johansson, "A Feasibility study of a capacitive biosensor for direct detection of DANN hybridization“, Vol. 11, Issue 3, pp. 156-160, 1999

16. J. Li, A. Cassel, L. Delzeit, J. Han, M. Meyyappan, "Novel threedimensional electrodes: electrochemical properties of carbon nanotube ensembles" J. Phys. Chem. B 2002, 106, 9299-9305

17. J.H. Chen, W.Z. Li, D.Z. Wang, S.X. Yang, J.G. Wen, Z.F., "electrochemical characterization of carbon nanotubes as electrode in electrochemical double-layer capacitors" Carbon 40 (2002) 1193-1197

18. J. Li, A. Cassel, L. Delzeit, J. Han, M. Meyyappan, "Novel threedimensional electrodes: electrochemical properties of carbon nanotube ensembles" J. Phys. Chem. B 2002, 106, 9299-9305

19. Sandro Carrara, Vijayender Kumar Bhalla, Claudio Stagni, Luca Benini, Anna Ferretti, Francesco Valle, Andrea Gallotta, Bruno Riccò, Bruno Samorì, Label-Free Cancer Markers Detection by Capacitance Biochip, Sensors and Actuators, 2008 submitted

20. D. Frank, U. Jaehde, U. Fuhr, Evaluation of probe drugs and pharmacokinetic metrics for CYP2D6 phenotyping, Eur J Clin Pharmacol (2007) 63:321-333 